\title{
BMJ Population-based cross-sectional study Open of barriers to utilisation of refraction services in South India: Rapid Assessment of Refractive Errors (RARE) Study
}

Srinivas Marmamula, ${ }^{1,2,3}$ Jill E Keeffe, ${ }^{2,4}$ Usha Raman,, ${ }^{1,5}$ Gullapalli N Rao ${ }^{1,3}$

To cite: Marmamula S, Keeffe JE, Raman U, et al. Population-based cross-sectional study of barriers to utilisation of refraction services in South India: Rapid Assessment of Refractive Errors (RARE) Study. BMJ Open 2011;1: e000172. doi:10.1136/ bmjopen-2011-000172

- Prepublication history for this paper is available online. To view these files please visit the journal online (http:// bmjopen.bmj.com).

Received 12 May 2011 Accepted 17 June 2011

This final article is available for use under the terms of the Creative Commons Attribution Non-Commercial 2.0 Licence; see http://bmjopen.bmj.com

${ }^{1}$ International Centre for Advancement of Rural Eye care, LV Prasad Eye Institute, Hyderabad, India

${ }^{2}$ Vision Cooperative Research Centre, Sydney, Australia

${ }^{3}$ Bausch \& Lomb School of Optometry, LV Prasad Eye Institute, Hyderabad, India ${ }^{4}$ Centre for Eye Research Australia, University of Melbourne, Melbourne, Australia

${ }^{5}$ Centre for Communication, LV Prasad Eye Institute, Hyderabad, India

Correspondence to Dr Srinivas Marmamula; srioptom@lvpei.org

\section{ABSTRACT}

Aim: To assess the barriers to the uptake of refraction services in the age group of 15-49 years in rural Andhra Pradesh, India.

Methods: A population-based cross-sectional study was conducted using cluster random sampling to enumerate 3300 individuals from 55 clusters. A validated questionnaire was used to elicit information on barriers to utilisation of services among individuals with uncorrected refractive error (presenting visual acuity $<6 / 12$ but improving to $\geq 6 / 12$ on using a pinhole) and presbyopia (binocular near vision $<\mathrm{N} 8$ in individuals aged $>35$ years with binocular distance visual acuity of $\geq 6 / 12$ ).

Results: 3095 (94\%) were available for examination. Those with uncorrected refractive errors cited affordability as the main barrier to the uptake of eye-care services. Among people with uncorrected presbyopia, lack of 'felt need' was the leading barrier. Conclusion: The barriers that were 'relatively easy to change' were reported by those with uncorrected refractive errors in contrast to 'difficult to change' barriers reported by those with uncorrected presbyopia. Together, the data on prevalence and an understanding of the barriers for the uptake of services are critical to the planning of refractive error services.

\section{INTRODUCTION}

Recent global estimates reveal that 153 million people have uncorrected refractive errors in addition to the 161 million people who are visually impaired due to other causes. ${ }^{1}$ Despite the availability of a simple remedy, uncorrected refractive errors are responsible for $16 \%$ of the blindness ${ }^{2}$ and $46 \%$ of the visual impairment across all age groups in the Indian state of Andhra Pradesh. ${ }^{3}$ Addressing the huge need for correction of uncorrected refractive error is one of the priorities of the global initiative VISION 2020: The Right to Sight. ${ }^{4}$

\section{ARTICLE SUMMARY}

Article focus

- Several barriers limit the uptake of refraction services.

- Understanding and addressing the barriers are essential to tackle the problem of uncorrected refractive errors and presbyopia.

Key messages

- Affordability is the important barrier among individuals with uncorrected refractive errors.

- Lack of felt need and awareness are important barriers reported by people with uncorrected presbyopia.

Strengths and limitations of this study

- A large sample size, good response rate and sound methodology are the strengths of the study.

- The study did not include individuals aged 50 and older, and hence the results cannot be generalised to the general population.

Uncorrected presbyopia is increasingly recognised as a major problem across the world. Recent estimates by Holden et a $\bar{l}$ have revealed that nearly 410 million people have near visual impairment due to uncorrected presbyopia. Several studies have underscored the impact of uncorrected presbyopia on the quality of life in individuals in rural settings. ${ }^{6-8}$ Recent studies make it clear that the impact of uncorrected presbyopia is not limited to literate populations living in urban areas.

Given the very limited data specifically focused on uncorrected refractive errors and presbyopia, especially in India, further research is necessary on barriers to the uptake of services. Understanding the perceived barriers is a prerequisite to formulating effective strategies to provide efficient and 
effective eye care. In this paper, we discuss the patientreported barriers to the uptake of refraction services among individuals aged 15-49 years living in rural areas of the South Indian state of Andhra Pradesh.

\section{MATERIALS AND METHODS}

The instrument that was used to collect information on barriers was part of a Rapid Assessment of Refractive Errors survey that was conducted in Mahbubnagar district in Andhra Pradesh, India. ${ }^{9}$ Five administrative divisions (mandals) in this district, each consisting of 20-30 villages, were selected and divided into clusters of almost equal population size. In total, 55 clusters were randomly selected using random numbers generated by an MS Excel worksheet, and attempts were made to examine 60 subjects from each cluster to obtain the sample size needed.

\section{Study procedures}

The survey team consisting of a vision technician (a high school graduate with 1 year's training in primary eye health) and two community eye-health workers visited subjects in their homes. Oral informed consent was sought from each subject after explaining the study and survey procedures. In the case of subjects aged below 18 years, permission was obtained from either the parent or guardian.

Presenting visual acuity (VA) in each eye was measured using a $\log$ MAR chart at a distance of $4 \mathrm{~m}$. Subjects with a VA of $<6 / 12(0.3 \log$ MAR $)$ in either eye were reassessed using a multiple pinhole occluder. Near vision was assessed binocularly using the $\mathrm{N}$ notation chart at the customary working distance (usual range $33-35 \mathrm{~cm}$ ) for each individual.

Demographic information including education level, occupation, current and previous use of spectacles was collected through a brief personal interview. The questionnaire used in this study had earlier been validated and used in the Andhra Pradesh Eye Disease Study. ${ }^{10}$ The response options in the questionnaire were derived from focus-group discussions held in the community ${ }^{11}$ and were used in previous studies. ${ }^{12} 13$ The questionnaire consisted of a list of 15 barriers. The questionnaire was administered in the regional languages (Telugu or Hindi) after the eye exam to those people with uncorrected refractive error and/or uncorrected presbyopia. If the response/s reported by the subject was on the list, then it was marked. In cases where one or more barriers reported were not in the list, they were fully specified under 'others.' If an individual gave more than one reason, all responses were marked, and the individual was asked to select the most important.

The barriers were then grouped under themes: (1) lack of awareness if the individual was unaware of uncorrected refractive errors or presbyopia; (2) lack of felt need if the individual was aware of uncorrected refractive errors or presbyopia but had never felt the need for consultation; (3) lack of affordability if the individual was aware of uncorrected refractive error and presbyopia but felt they could not afford the cost of eye exam and/or spectacles; (4) lack of accessibility if the individual stated that services were too far away or difficult to reach; (5) personal barrier if the individual mentioned other health-related problems, fear or others such as emotional and psychological issues.

\section{Study definitions}

A barrier was defined as the reason for not accessing an eye-care facility by persons who could benefit from spectacles. Visual impairment was defined as binocular presenting VA $<6 / 12$. Uncorrected refractive error was defined as presenting VA $<6 / 12$ but improving to $\geq 6 / 12$ on using a pinhole. Uncorrected presbyopia was defined as binocular near vision $<\mathrm{N} 8$ at the subject's customary working distance in subjects aged $>35$ years and who had a binocular presenting distance VA of 6/12 (logMAR 0.3 ) or better. It was classified as mild (presenting near vision $<\mathrm{N} 8$ to $\mathrm{N} 10$ ), moderate (worse than N10 to N18) and severe $(<\mathrm{N} 18)$.

Data management and analysis were conducted using SPSS V.16.0 software. All persons with VA $<6 / 12$ in the either eye were referred to the nearest eye-care facility for management. The survey was conducted in accordance with ethical principles and adhered to the Declaration of Helsinki. This study was carried out during February-May 2008. The study protocol was reviewed and approved by the Institutional Review Board of LV Prasad Eye Institute, Hyderabad, India.

\section{RESULTS}

Of the 3300 subjects enumerated from 55 clusters, the data were available from 3095 subjects $(94 \%)$ for analysis. The mean ages of males and females were similar $(p=0.34)$, and over half the participants had no education. The participants' characteristics are presented in table 1 .

\section{Uncorrected refractive errors}

Refractive errors were present in either eye of 187 subjects (age and gender-adjusted prevalence of $4.8 \%$, $95 \%$ CI $4.0 \%$ to $5.5 \%$ ), and the condition was uncorrected in 139 subjects. Of these 139 individuals, $30.9 \%$ (43 subjects) cited an economic reason as a barrier to the uptake of services, and 23.0\% (32 subjects) cited a lack of 'felt need' for the refractive correction. Another $16.5 \%$ (23) cited 'lack of access' as the barrier for uptake of services (table 2). The barriers were similar among the individuals who had uncorrected refractive errors in one or both eyes (table 2). In 88 $(2.8 \%)$ subjects, uncorrected refractive errors were present in the better eye and were the cause of visual impairment. An economic reason followed by 'lack of access' were reported as the most important barriers in this group (table 2).

Out of 139 subjects with uncorrected refractive errors in either eye, 63 individuals gave more than one response. Personal reasons and lack of 'felt need' were the most common additional barriers. Similar barriers 


\begin{tabular}{lc} 
Table 1 Participants' characteristics $(\mathrm{n}=3095)$ \\
\hline \multicolumn{2}{c}{$\mathbf{n}(\%)$} \\
\hline Age group (years) \\
15-29 \\
$30-39$ & $1540(49.8)$ \\
$40-49$ & $739(23.9)$ \\
Gender & $816(26.4)$ \\
Male & \\
Female & $1626(52.5)$ \\
Education level & $1469(47.5)$ \\
No education & \\
School education & $1794(58.0)$ \\
University education & $1095(35.4)$ \\
Occupation & $206(6.7)$ \\
Unskilled labour & \\
Home duties & $1722(55.6)$ \\
Student & $557(18.0)$ \\
Skilled jobs & $392(12.7)$ \\
Clerical/business & $227(7.3)$ \\
\end{tabular}

were reported by 45 individuals with uncorrected refractive errors in the better eye and who gave more than one response (table 2).

\section{Uncorrected presbyopia}

Among the 974 subjects aged above 35 years, presbyopia was present in 616 (63.2\%; 95\% CI 60.2 to 66.2$)$ subjects. It was uncorrected in 512/616 (83.1\%) subjects. Lack of 'felt need' was the most important barrier to the uptake of services in $46.4 \%$ (237) of individuals. Lack of 'awareness' was reported by $16 \%$ (82) participants. 'Lack of access,' economic and personal reasons were reported by $13 \%, 12.5 \%$ and $11.7 \%$ respectively (table 3 ).

The lack of 'felt need' was the main barrier irrespective of severity of uncorrected presbyopia: $50.2 \%$ (117 subjects) with mild and $43.2 \%$ (120 subjects) with moderate presbyopia (table 3 ). 'Lack of accessibility' to services as a barrier was higher among the subjects with moderate presbyopia compared with those with mild presbyopia $\left(16 \%\right.$ vs $9 \%, \chi^{2}$ test, $\left.p=0.009\right)$. Lack of awareness was higher in mild presbyopes compared with moderate presbyopes (23\% and $10 \%$ respectively, $\chi^{2}$, $\mathrm{p}=0.001)$. Of 512 subjects with uncorrected presbyopia, 201 individuals gave more than one response. Lack of 'felt need' and personal reasons were the leading barriers in this group (table 3 ).

\section{DISCUSSION}

The utilisation of refraction services is just as important as provision of services, if we were to address the burden of uncorrected refractive errors and presbyopia. To our knowledge, this is the first study to report barriers to the uptake of refraction services from India that has implications for the planning and implementation of eye care services. The barriers reported by the individuals studied can be categorised as 'relatively easy to change' and 'difficult to change.' For example, economic barriers and accessibility-related barriers may be categorised as relatively easy to change. The initiative to change this is with the service provider, by making services more affordable and more easily reachable in terms of location. Lack of 'felt need,' awareness and personal reasons such as fear and other competing commitments are more difficult to change, and a sustained long-term effort is required both at the individual level and by the service provider to create an impact. Related to these personal barriers are issues such as differential access among women and older people, due to cultural barriers or because of a lack of supportive family structure. Though these were not specifically included in the list, they are definitely barriers to consider and perhaps to some extent overlap with other personal barriers.

For uncorrected refractive errors, the most important barriers are 'easy to change' but importantly almost half of the participants $(63 / 139)$ reported more than one barrier, most of which were in the 'difficult to change' category. In contrast to this, 'difficult to change' are the leading barriers for uncorrected presbyopia. Even among those reporting a second barrier, 'difficult to change' barriers are the most important. These findings reflect the need for a rigorous campaign to address the benefits of correction of presbyopia.

Refraction services are provided mainly at the primary and, to some extent, at the secondary level of eye care in India. The study highlights economic reasons as the leading barrier for uptake of services for correction of refractive errors and a lack of 'felt need' as the leading barrier for uncorrected presbyopia. Economic barriers

Table 2 Barriers and uncorrected refractive errors (URE) in either eye or the better eye

\begin{tabular}{|c|c|c|c|c|}
\hline & \multicolumn{2}{|l|}{ URE either eye } & \multicolumn{2}{|l|}{ URE in better eye } \\
\hline & $\begin{array}{c}\mathrm{n}(\%) \\
\text { Most important barrier }\end{array}$ & $\begin{array}{c}\mathrm{n}(\%) \\
\text { Second barrier }\end{array}$ & $\begin{array}{c}\mathrm{n}(\%) \\
\text { Most important barrier }\end{array}$ & $\begin{array}{c}\mathrm{n}(\%) \\
\text { Second barrier }\end{array}$ \\
\hline Economic reasons & $43(30.9)$ & $8(12.7)$ & $32(36.4)$ & $6(13.3)$ \\
\hline Lack of felt need & $32(23.0)$ & $11(17.5)$ & $21(23.9)$ & 7 (15.6) \\
\hline Lack of access & $23(16.5)$ & $7(11.1)$ & $12(13.6)$ & $4(8.9)$ \\
\hline Personal reasons & $23(16.5)$ & $29(46.0)$ & $12(13.6)$ & $23(51.1)$ \\
\hline Lack of awareness & 18 (12.9) & 8 (12.7) & $11(12.5)$ & $5(11.1)$ \\
\hline Total & $139(100.0)$ & $63(100.0)$ & $88(100.0)$ & $45(100.0)$ \\
\hline
\end{tabular}


Table 3 Barriers and uncorrected presbyopia

\begin{tabular}{|c|c|c|c|c|}
\hline & \multicolumn{3}{|c|}{ Most important barrier } & \multirow{2}{*}{$\begin{array}{l}\text { Second barrier } \\
\text { Total presbyopia* } \\
\text { n (\%) }\end{array}$} \\
\hline & $\begin{array}{l}\text { Mild presbyopia } \\
\text { n (\%) }\end{array}$ & $\begin{array}{l}\text { Moderate presbyopia } \\
\text { n (\%) }\end{array}$ & $\begin{array}{l}\text { Total presbyopia* } \\
\text { n (\%) }\end{array}$ & \\
\hline Lack of felt need & $117(50.2)$ & $120(43.2)$ & $237(46.4)$ & $50(24.9)$ \\
\hline Lack of awareness & $53(22.7)$ & $29(10.4)$ & $82(16.0)$ & $40(19.9)$ \\
\hline Economics & $22(9.4)$ & $42(15.1)$ & $64(12.5)$ & 28 (13.9) \\
\hline Accessibility & $21(9.0)$ & 47 (16.9) & 68 (13.3) & $39(19.4)$ \\
\hline Personal reasons & $20(8.6)$ & $40(14.4)$ & $60(11.7)$ & $44(21.9)$ \\
\hline Total & $233(100.0)$ & $278(100.0)$ & $511(100.0)$ & $201(100.0)$ \\
\hline
\end{tabular}

include cost of a consultation and the cost of spectacles. In the context of the study, refraction services are often provided free of charge, and a patient needs to pay for spectacles. Hence, economic barriers are mainly related to the cost of spectacles. Several studies from developing countries, including those by Nirmalan and colleagues from South India, ${ }^{14}$ and Kovai and colleagues who found that $37 \%$ of those who noticed a change in vision did not utilise eye-care services for economic reasons in rural Andhra Pradesh, ${ }^{13}$ have identified economic reasons as an important barrier for uptake of services. $^{15-17}$

Provision of spectacles at a low cost and an affordable pricing system would address the issue of uncorrected refractive errors and presbyopia. If the cost of spectacles is high, it may be unaffordable to many people. Hence, the pricing system should reflect the purchasing capacity of the majority in the community so as to encourage the sale of spectacles. Anecdotal evidence in these rural communities suggests that an individual may be able to afford 2 days' wages (approximately 150-200 Indian rupees or US\$4-5) to buy a pair spectacles.

In addition to affordability, the quality, comfort, endurance and accuracy of the prescription are equally important. Dandona et $a l^{18}$ found that nearly one-third of the subjects with correctable visual impairment discontinued the use of spectacles, either because they felt the prescription was wrong or because they felt the spectacles were uncomfortable. There are similar reports from Timor-Leste. ${ }^{16}$

In the current study, a quarter of those with uncorrected refractive errors did not feel the need for correction, possibly because they did not face problems in their day-to-day tasks. Although other barriers cannot be ignored, the lack of a felt need for correction of refractive errors is important in considering targets for the elimination of uncorrected refractive errors. Setting targets purely based on prevalence estimates from epidemiological studies, without discounting for those who do not feel the need for correction, may be difficult to achieve. Further, it is useful to understand this aspect in greater depth, by probing, for instance, the social and cultural factors that lead to someone not 'feeling' the need for vision correction, or how 'good vision' is understood by different groups of people.
Only a few studies have referred to the prevalence and impact of presbyopia from the developing world. ${ }^{6-8}$ In the present study, a 'lack of felt need' followed by a 'lack of awareness' is a major barrier to the uptake of services, among people with presbyopia. Together, they accounted for over $60 \%$ of the responses. Sherwin et al concluded that a quarter of the subjects with presbyopia in a rural Kenyan population did not consider their condition to be important. ${ }^{19}$ Nirmalan et al reported that about $24 \%$ of the subjects did not consider presbyopia to be a serious problem, and another $24 \%$ of them felt that they were able to see adequately. ${ }^{20}$

Lack of awareness was cited frequently by the presbyopic population in the current study, which did not include individuals aged 50 years and above as in other studies in presbyopia. Here, this was significantly higher in those with mild presbyopia than the moderate presbyopia group. Lack of access is reported to be more common for those with moderate presbyopia compared with mild presbyopia. As near vision decreases to a level where it affects daily routine, people seem more eager to use services, and at this point accessibility becomes a crucial factor.

Extrapolating the results from this study, even if we consider a conservative estimate of $40 \%$ of the 410 million people globally reported to have uncorrected presbyopia $^{5}$ have 'felt need' as a barrier for near correction, the target for service delivery will decrease considerably to 246 million. It is essential to consider these barriers for planning and setting of targets for refraction services. Planning based on the total need as estimated by a service provider, instead of patient 'perceived need,' is bound to overestimate the target by a fair margin. 'Felt need' drives the demand for presbyopic spectacles. In this study, about $58 \%$ of the subjects had no formal education, and $56 \%$ were involved in unskilled labour. It may be inferred that in regions where the level of education is high, the felt need may be higher, and similarly in areas where a majority of people are engaged in near-work-related occupations, the high felt need may be seen. Even though the study did not include severe presbyopes and was conducted in rural areas, the estimates may be skewed but could have a considerable effect in similar populations. 
Other issues related to affordability and availability are also important. For instance, if services are easily available and affordable, the effort an individual has to make to obtain a pair of spectacles is lower, and this may increase the uptake of services. This uptake may be low if the individual has to travel long distances and spend more money on direct or indirect costs to procure the same pair of spectacles. It may also be influenced by the dynamics in the family, culture and community; for instance, gender has been cited as a barrier in general to accessibility of services. ${ }^{21} 22$ Individuals who cannot draw upon the support of family or care givers to accompany them to the clinic or to provide related assistance may be less likely to act upon a need when it is felt.

The recent strategy of provision of eye-care services, mainly refraction services through permanent facilities called vision centres, is a step in the right direction to address several barriers reported in this paper. ${ }^{23} 24$ Vision centres are primary eye-care units staffed by a 'vision technician,' located strategically to maximise access to underserved communities. Each vision centre is designed to cater for the primary eye-care needs of 50000 population and forms three core functions (three Rs) (1) recognise common blinding conditions; (2) refraction and dispensing of spectacles at a low cost; and (3) referral services, if a patient needs a further eye examination for medical and surgical intervention.

To conclude, the results of the study, using Rapid Assessment of Refractive Errors methodology, revealed several barriers to utilisation of refraction services in Mahbubnagar district in Andhra Pradesh, India. Together, the data on prevalence and understanding and addressing the barriers for the uptake of services are critical to the planning of refractive error services in this region and can help to achieve the overall goals of the VISION 2020 initiative.

Acknowledgements The authors thank the volunteers who have participated in the study. The authors also thank the vision technicians, S Narasiah and $\mathrm{G}$ Bhaskar, for their assistance in data collection.

Funding The financial support for this study was provided in part by the Vision Co-operative Research Centre, Australia as part of SM's doctoral programme and by Hyderabad Eye Research Foundation. GNR and JEK supervised the project and provided technical inputs. UR reviewed the earlier drafts of the manuscripts and provided inputs.

Competing interests None.

Ethics approval Institutional Review Board of LV Prasad Eye Institute.

Contributors SM conceived the idea, and planned and implemented the study; JEK helped in designing the study protocol, supervised the project and contributed in interpreting the data, writing and revising the manuscript; UR and GNR helped in interpreting the data, reviewed the earlier and revised versions of the manuscript. All authors approved the final version of the manuscript.

Provenance and peer review Not commissioned; externally peer reviewed.

Data sharing statement No additional data available.

\section{REFERENCES}

1. Resnikoff S, Pascolini D, Mariotti SP, et al. Global magnitude of visual impairment caused by uncorrected refractive errors in 2004. Bull World Health Organ 2008;86:63-70.

2. Dandona $L$, Dandona $R$, Srinivas $M$, et al. Blindness in the Indian state of Andhra Pradesh. Invest Ophthalmol Vis Sci 2001;42:908-16.

3. Dandona R, Dandona L, Srinivas M, et al. Moderate visual impairment in India: the Andhra Pradesh Eye Disease Study. Br J Ophthalmol 2002;86:373-7.

4. WHO. Elimination of Avoidable Visual Disability Due to Refractive Errors, in WHO/PBL/00.79. Geneva: World Health Organization, 2001.

5. Holden BA, Fricke TR, Ho SM, et al. Global vision impairment due to uncorrected presbyopia. Arch Ophthalmol 2008;126:1731-9.

6. Burke AG, Patel I, Munoz B, et al. Population-based study of presbyopia in rural Tanzania. Ophthalmology 2006;113:723-7.

7. Patel I, Munoz B, Burke AG, et al. Impact of presbyopia on quality of life in a rural African setting. Ophthalmology 2006;113:728-34.

8. Lu Q, He W, Murthy GV, et al. Presbyopia and near-vision impairment in rural northern China. Invest Ophthalmol Vis Sci 2011;52:2300-5.

9. Marmamula S, Keeffe JE, Rao GN. Uncorrected refractive errors, presbyopia and spectacle coverage: results from a rapid assessment of refractive error survey. Ophthalmic Epidemiol 2009;16:269-74.

10. Dandona R, Dandona L, Naduvilath TJ, et al. Design of a populationbased study of visual impairment in India: The Andhra Pradesh Eye Disease Study. Indian J Ophthalmol 1997;45:251-7.

11. Dandona R, Dandona L. Review of findings of the Andhra Pradesh Eye Disease Study: policy implications for eye-care services. Indian J Ophthalmol 2001;49:215-34.

12. Dandona R, Dandona L, Naduvilath TJ, et al. Utilisation of eyecare services in an urban population in southern India: the Andhra Pradesh eye disease study. Br J Ophthalmol 2000;84:22-7.

13. Kovai V, Krishnaiah S, Shamanna BR, et al. Barriers to accessing eye care services among visually impaired populations in rural Andhra Pradesh, South India. Indian J Ophthalmol 2007;55:365-71.

14. Nirmalan PK, Katz J, Robin AL, et al. Utilisation of eye care services in rural south India: the Aravind Comprehensive Eye Survey. $\mathrm{Br} \mathrm{J}$ Ophthalmol 2004;88:1237-41.

15. Hodges LE, Berk ML. Unmet need for eyeglasses: results from the 1994 Robert Wood Johnson Access to Care Survey. J Am Optom Assoc 1999;70:261-5.

16. Ramke J, du Toit R, Palagyi A, et al. Correction of refractive error and presbyopia in Timor-Leste. Br J Ophthalmol 2007;91:860-6.

17. Yasmin $\mathrm{S}$, Minto $\mathrm{H}$. Community perceptions of refractive errors in Pakistan. Community Eye Health 2007;20:52-3.

18. Dandona R, Dandona L, Kovai V, et al. Population-based study of spectacles use in southern India. Indian J Ophthalmol 2002;50:145-55.

19. Sherwin JC, Keeffe JE, Kuper $\mathrm{H}$, et al. Functional presbyopia in a rural Kenyan population: the unmet presbyopic need. Clin Exp Ophthalmol 2008;36:245-51.

20. Nirmalan PK, Krishnaiah S, Shamanna BR, et al. A population-based assessment of presbyopia in the state of Andhra Pradesh, south India: the Andhra Pradesh Eye Disease Study. Invest Ophthalmol Vis Sci 2006;47:2324-8.

21. Courtright P. Gender and blindness: Taking a global and a local perspective. Oman J Ophthalmol 2009;2:55-6.

22. Courtright P. Bassett K. Gender and blindness: eye disease and the use of eye care services. Community Eye Health 2003;16:11-12.

23. Khanna R, Raman U, Rao GN. Blindness and poverty in India: the way forward. Clin Exp Optom 2007;90:406-14.

24. Rao GN. An infrastructure model for the implementation of VISION 2020: the right to sight. Can J Ophthalmol 2004;39:589-94. 